\title{
Association of Physical Activity with Lipodystrophy Syndrome in HIV- Infected Patients
}

Fabiana Schuelter Trevisol ${ }^{1,2}$, Paulo R Alencastro ${ }^{3}$, Paula AB Ribeiro ${ }^{1}$, Fernando H Wollf ${ }^{3}$, Maria Letícia R Ikeda ${ }^{1}$, Nêmora T Barcellos ${ }^{3}$, Ajácio BM Brandão ${ }^{3}$ and Sandra C Fuchs ${ }^{1,3^{*}}$

${ }^{1}$ Postgraduate Studies Program in Cardiology, School of Medicine, Universidade Federal do Rio Grande do Sul, R. Ramiro Barcelos 2600, CEP 90035-003, Porto Alegre, RS, Brazil

${ }^{2}$ Postgraduate Program-Master of Health Sciences, Universidade do Sul de Santa Catarina, Brazil

${ }^{3}$ Division of Cardiology, and the National Institute for Science and Technology for Health Technology Assessment (IATS/CNPq), Hospital de Clínicas de Porto Alegre. R. Ramiro Barcelos 2350, Centro de Pesquisas, Cardiolab-Hipertensão, CEP 90035-003, Porto Alegre, RS, Brazil

\begin{abstract}
Highly Active Antiretroviral Therapy (HAART) has increased life expectancy of HIV-infected patients, but may also increase triglyceride and cholesterol levels, triggering lipodystrophy syndrome. Physical activity may prevent or attenuate such adverse effects, but it has not been fully evaluated in HIV-infected patients. This cross-sectional study aimed to investigate the association between physical activity and lipodystrophy syndrome in HIV-infected individuals, 18 years or older. Physical activity was evaluated by the short version of the International Physical Activity Questionnaire. Lipodystrophy was verified by at least two reporting of changes in different parts of the body, or directly assessed, categorized as lipoatrophy or lipohypertrophy. Among 1,240 participants, $46 \%$ had lipohypertrophy, which was independently associated with insufficient physical activity in men, but not in women. The prevalence of lipoatrophy was $53.2 \%$. Metabolic parameters were higher among individuals on HAART, in comparison to HAART-naive patients. In conclusion, HAART-naive physically active individuals had lower metabolic profile than among insufficiently active.
\end{abstract}

Keywords: HIV/AIDS; Lipodystrophy; Physical activity; Exercise; Lifestyle

\section{Introduction}

Highly Active Antiretroviral Therapy (HAART) has significantly changed the morbidity and quality of life among HIV-infected patients [1]. However, HIV-associated lipodystrophy syndrome is among the adverse effects of HAART, characterized by atrophy in the peripheral regions (fat reduction in the face, arms and legs) and lipohypertrophy in the central region (fat accumulation in the chest, abdomen, neck and dorsocervical region) [2-4], which can be presented alone or combined (mixed lipodystrophy) [2,3]. The syndrome may also increase the levels of triglycerides, total cholesterol, Low-Density Lipoprotein fraction (LDL) and glucose, and reduce the levels of High-Density Lipoprotein (HDL) [4,5].

Although HIV-associated lipodystrophy syndrome is clearly influenced by the use of HAART, reverse transcriptase inhibitors are strongly associated with lipoatrophy and protease inhibitors to lipohypertrophy and lipid disorders. The pathophysiology of HIVassociated lipodystrophy syndrome is not yet fully elucidated though. Factors associated with lipodystrophy include gender, age, class of antiretroviral and duration of HAART use [6]. There are reports of these changes among HIV-individuals that are not under antiretroviral treatment [4]. Thus, AIDS is now classified as a chronic disease, resulting in increased cardiovascular risk [7].

Behavioral changes and lifestyle, such as diet and physical activity (and/or exercise), smoking cessation and reduction of alcohol consumption could be non-pharmacological strategies for the prevention or treatment of HIV lipodystrophy syndrome. The prevalence of smoking is high among HIV-infected persons [8], and this habit causes serious health problems, such as cardiorespiratory disorders that can accelerate the progression to AIDS, i.e., the installation of opportunistic diseases [9], reducing levels of CD4 count and viral load increase $[10,11]$. Healthy lifestyle habits are important for the general population and are also relevant for HIV-infected individuals [8]. However, exercise can worsen lipoatrophy $[2,6]$, even though it has beneficial effects on lipohypertrophy and lipid levels [9-12]. Generally, among people infected with HIV or not, there are reports on the fact that women have a higher accumulation of abdominal fat, and men are more physically active, which is why the data should be analyzed by gender $[13,14]$.

This study aimed to investigate the association between physical activity and HIV-associated lipodystrophy syndrome and metabolic profile.

\section{Methods}

A cross-sectional study enrolled HIV-infected male and female patients, aged 18 years or older, between 2006 and 2008, attending a secondary hospital, which is a reference center for HIV-testing and outpatient treatment for HIV, in Porto Alegre, southern Brazil. Patients under the influence of illicit drugs or alcohol at the time of the interview were excluded, as well as pregnant women and subjects with participation restrictions due to mental disabilities or restraints

*Corresponding author: Sandra C Fuchs, Centro de Pesquisa Clínica CARDIOLAB-Hipertensão Hospital de Clínicas de Porto Alegre, Universidade Federal do Rio Grande do Sul, Ramiro Barcellos, 2350, $5^{\circ}$ andar, 90.035-000 Porto Alegre, RS, Brazil, Tel: + 5551-33597621; Fax: + 5551-3359-8420; E-mail: scfuchs@terra.com.br

Received August 28, 2012; Accepted October 18, 2012; Published October 27 2012

Citation: Trevisol FS, Alencastro PR, Ribeiro PAB, Wollf FH, Ikeda MLR, et al. (2012) Association of Physical Activity with Lipodystrophy Syndrome in HIV-Infected Patients. J AIDS Clinic Res 3:177. doi:10.4172/2155-6113.1000177

Copyright: (c) 2012 Trevisol FS, et al. This is an open-access article distributed under the terms of the Creative Commons Attribution License, which permits unrestricted use, distribution, and reproduction in any medium, provided the original author and source are credited. 
of freedom. The Institution Review Board approved the protocol and patients signed an informed consent to participate.

\section{Study enrollment and definitions}

Patients were interviewed using a standardized questionnaire during the routine visit for HIV treatment, including questions pertaining to demographic (age, gender, race), socioeconomic (education) and lifestyle (physical activity, smoking and alcohol consumption) characteristics by certified interviewers. Clinical data (use of highly active antiretroviral therapy and duration of HIV-infection) and laboratory information (CD4 count and HIV viral load) were obtained from medical records, for the three months prior to the interview. Patients were considered on highly active antiretroviral therapy (HAART) use, if they were taking three antiretroviral drugs; naive-treatment patients were those who had never used any antiretroviral drug. Viral load below 50 copies $/ \mathrm{mL}$ HIV RNA was considered undetectable. Race was self-reported and participants were categorized as Whites or non-Whites. Education was measured by years of schooling, categorized as high school education (yes or no). Smokers were characterized by lifetime consumption of at least 100 cigarettes, and pack-year of smoking was calculated by multiplying the number of packs smoked per day by the number of years smoked [15].

Weight, height, waist circumference, hip, arm and neck, and three facial skinfolds-infraorbital, submandibular and buccal-measurements were performed in duplicate and the average was used for analysis. Body circumference measurements were performed using inelastic anthropometric tape. The midpoint between the anterior superior iliac spine and iliac crest and the inferior costal rib was used for waist circumference. Hip circumference was measured at its largest area. Neck circumference was performed with the patient sitting, with the area uncovered, positioning the tape over the Adam's apple. Circumference of the arm was measured at the midpoint between the acromion and olecranon, with the limb positioned at 90 degrees. Facial skinfolds (infraorbital, buccal and submandibular) were measured using a Cescorf brand adipometer.

To identify alcohol consumption abuse, even occasional, binge drinking category was created for individuals with intake of at least five drinks of any alcoholic beverage on a single occasion.

Inquiries on self-awareness of body changes after HIV infection diagnosis were made. The questions were asked to determine whether the patients had noticed changes in body fat distribution, increase or decrease in general and in specific parts of the body.

Lipodystrophy was determined by the presence of at least two changes in different parts of body, using self-perception and objective measurements. Self-perception of body changes was based on increase or decrease of body fat, or on body parts, including face, dorsocervical fat pads, stomach, chest or breasts; or on the reduction of arms, legs, waist, buttocks, and sunken cheeks in the face. Objective measurements included neck, waist, and hip circumferences, and skin-fold thicknesses on the face. Lipohypertrophy and lipoatrophy were determined by a combination of at least two measurements at $90^{\text {th }}$ and $10^{\text {th }}$ percentiles, respectively, and/or positive signs based on patient's self-perception.

Patients for whom information lipid profile-total cholesterol, High Density Lipoprotein (HDL) fraction and Low Density Lipoprotein (LDL), triglycerides and fasting glucose-could not be obtain from medical records of the examinations performed over the last two months, were given request for laboratory examinations. Blood sample was collected after a 12-hour fasting period, and all examinations were performed at the same clinical laboratory.

\section{Physical activity}

Physical activity was evaluated by the short version of the International Physical Activity Questionnaire (IPAQ), and participants were categorized as active if they had performed $\geq 150$ minutes of moderate to vigorous intensity physical activity along five days in the previous week or as insufficiently active otherwise.

\section{Statistics}

Epi Info version 3.5.1 (Centers for Disease Control) was used for double data entry. Statistical analysis was performed using the Statistical Package for Social Sciences (SPSS for Windows v 16; Chicago, IL, USA). Prevalence ratio $(95 \% \mathrm{CI})$ was calculated for characteristics associated with physical activity, crude and subsequently adjusted for potential confounding variables, using modified Poisson regression models. Confounding factors were selected among variables associated with physical activity, in the bivariate analysis ( $P$ value $<0.10$ ), or those described as such in the literature (Age, education and alcohol consumption abuse).

\section{Results}

A total of 1,240 HIV-infected patients were enrolled; mean age was $39.1 \pm 10.1$ years; $50.6 \%$ were men, aged $40 \pm 9.6$ years, $49.4 \%$ women, whose average age was $38.2 \pm 10.4$ years.

The prevalence of lipoatrophy was $53.2 \%$ and lipohypertrophy was 46\% among HIV-infected individuals.

The characteristics of the participants, classified according to the presence or absence of lipohypertrophy, are shown in table 1 .

Of the total study participants, females, non-Whites, non-smokers,

\begin{tabular}{|c|c|c|c|c|}
\hline & \multirow{2}{*}{$\begin{array}{l}\text { Total } \\
(n=1,240)\end{array}$} & \multicolumn{3}{|c|}{ Prevalence of Lipohypertrophy } \\
\hline & & $\begin{array}{l}\text { Overall } \\
(n=1240)\end{array}$ & $\begin{array}{l}\text { Men } \\
(n=628)\end{array}$ & $\begin{array}{l}\text { Women } \\
(n=612)\end{array}$ \\
\hline \multicolumn{5}{|l|}{ Gender } \\
\hline Men & 628 (50.6) & $242(38.5)$ & - & - \\
\hline Women & $612(49.4)$ & $328(53.6)$ & - & - \\
\hline$P$ value & & $<0.001$ & & \\
\hline \multicolumn{5}{|l|}{ Age (years) } \\
\hline $18-34$ & $471(38.0)$ & $211(44.8)$ & $69(32.7)$ & $142(67.3)$ \\
\hline $35-49$ & 591 (47.7) & 266 (45.0) & $135(50.8)$ & 131 (49.2) \\
\hline $50-78$ & $178(14.3)$ & $93(52.2)$ & $38(40.9)$ & 55 (59.1) \\
\hline$P$ value & & 0.2 & 0.3 & 0.3 \\
\hline \multicolumn{5}{|l|}{ Skin color } \\
\hline White & $692(55.8)$ & $298(43.1)$ & $148(49.7)$ & $150(50.3)$ \\
\hline Non-white & $548(44.2)$ & $272(49.6)$ & $94(34.6)$ & $178(65.4)$ \\
\hline$P$ value & & 0.02 & 0.8 & 0.005 \\
\hline \multicolumn{5}{|l|}{ Years at school } \\
\hline$<12$ & 1,097 (88.5) & 504 (45.9) & 199 (39.5) & 305 (60.5) \\
\hline$\geq 12$ & $143(11.5)$ & $66(46.2)$ & $43(65.2)$ & $23(34.8)$ \\
\hline$P$ value & & 1.0 & 0.17 & 0.5 \\
\hline \multicolumn{5}{|c|}{ Smoking (pack-years) } \\
\hline Never-smoker & $422(34.0)$ & $221(52.4)$ & $81(36.7)$ & $140(63.3)$ \\
\hline$<20$ & 498 (40.2) & $211(42.4)$ & $87(41.2)$ & $124(58.8)$ \\
\hline$\geq 20$ & $320(25.8)$ & $138(43.1)$ & $74(53.6)$ & $64(46.4)$ \\
\hline$P$ value & & 0.005 & 0.15 & 0.06 \\
\hline \multicolumn{5}{|l|}{ Physical activity } \\
\hline Active & 716 (57.7) & 324 (45.3) & 114 (35.2) & $210(64.8)$ \\
\hline
\end{tabular}


Citation: Trevisol FS, Alencastro PR, Ribeiro PAB, Wollf FH, Ikeda MLR, et al. (2012) Association of Physical Activity with Lipodystrophy Syndrome in HIV-Infected Patients. J AIDS Clinic Res 3:177. doi:10.4172/2155-6113.1000177

Page 3 of 7

\begin{tabular}{|c|c|c|c|c|}
\hline Inactive or sedentary & $524(42.3)$ & $246(46.9)$ & $128(52.0)$ & $118(48.0)$ \\
\hline$P$ value & & 0.6 & 0.008 & 0.2 \\
\hline \multicolumn{5}{|l|}{ Binge drinking } \\
\hline Yes & $211(17.0)$ & $91(43.1)$ & $56(61.5)$ & $35(38.5)$ \\
\hline No & $1,029(83.0)$ & $479(46.6)$ & $186(38.8)$ & $293(61.2)$ \\
\hline$P$ value & & 0.6 & 0.9 & 0.8 \\
\hline \multicolumn{5}{|l|}{ Body mass index $\left(\mathrm{kg} / \mathrm{m}^{2}\right)$} \\
\hline$<25$ & $708(57.1)$ & $223(31.5)$ & $94(42.2)$ & $129(57.8)$ \\
\hline $25-29.9$ & $380(30.6)$ & $220(57.9)$ & $105(47.7)$ & $115(52.3)$ \\
\hline$\geq 30$ & $152(12.3)$ & $127(83.6)$ & $43(33.9)$ & $84(66.1)$ \\
\hline$P$ value & & $<0.001$ & $<0.001$ & $<0.001$ \\
\hline \multicolumn{5}{|c|}{ Years since HIV diagnosis } \\
\hline$<3$ & $485(39.1)$ & $206(42.5)$ & $82(39.8)$ & $124(60.2)$ \\
\hline $3-5$ & $350(28.3)$ & $169(48.3)$ & $73(43.2)$ & $96(56.8)$ \\
\hline$\geq 6$ & $404(32.6)$ & $195(48.3)$ & $87(44.6)$ & $108(55.4)$ \\
\hline$P$ value & & 0.14 & 0.19 & 0.4 \\
\hline \multicolumn{5}{|l|}{ Currently on HAART } \\
\hline Yes & $815(65.7)$ & $409(50.2)$ & $167(40.8)$ & $242(59.2)$ \\
\hline No & $425(34.3)$ & $161(37.9)$ & $75(46.6)$ & $86(53.4)$ \\
\hline$P$ value & & $<0.001$ & 0.4 & $<0.001$ \\
\hline \multicolumn{5}{|c|}{ CD4 count $\left(\mathrm{cel} / \mathrm{mm}^{3}\right)(\mathrm{n}=1,227)$} \\
\hline$<350$ & $476(38.8)$ & $193(40.5)$ & $86(44.6)$ & $107(55.4)$ \\
\hline$\geq 350$ & $751(61.2)$ & $372(49.5)$ & $153(41.1)$ & $219(58.9)$ \\
\hline$P$ value & & 0.002 & 0.06 & 0.03 \\
\hline \multicolumn{5}{|c|}{ HIV-RNA viral load $(n=1,221)$} \\
\hline Undetectable & $713(57.5)$ & $280(39.3)$ & $124(44.3)$ & $156(55.7)$ \\
\hline Detectable & $508(42.5)$ & $282(55.5)$ & $113(40.1)$ & $169(59.9)$ \\
\hline$P$ value & & $<0.001$ & $<0.001$ & $<0.001$ \\
\hline \multicolumn{5}{|c|}{ Total cholesterol $(\mathrm{mg} / \mathrm{dL})(\mathrm{n}=1,226)$} \\
\hline$<200$ & $854(69.7)$ & $352(41.2)$ & $148(42.0)$ & $204(58.0)$ \\
\hline$\geq 200$ & $372(30.3)$ & $214(57.5)$ & $90(42.1)$ & $124(57.9)$ \\
\hline$P$ value & & $<0.001$ & $<0.001$ & 0.001 \\
\hline \multicolumn{5}{|l|}{$\mathrm{HDL}(\mathrm{mg} / \mathrm{dL})(\mathrm{n}=1,226)$} \\
\hline$<35$ & $152(12.4)$ & $50(32.9)$ & $28(56.0)$ & $22(44.0)$ \\
\hline$\geq 35$ & $\begin{array}{l}1,074(87.6) \\
(86.7)\end{array}$ & $516(48.0)$ & $210(40.7)$ & $306(59.3)$ \\
\hline$P$ value & & $<0.001$ & 0.006 & 0.3 \\
\hline \multicolumn{5}{|l|}{$\operatorname{LDL}(m g / d L)(n=1,225)$} \\
\hline$<130$ & 966 (78.9) & $424(43.9)$ & $188(44.3)$ & $236(55.7)$ \\
\hline$\geq 130$ & $259(21.1)$ & $142(54.8)$ & $50(35.2)$ & $92(64.8)$ \\
\hline$P$ value & & 0.002 & 0.04 & 0.1 \\
\hline \multicolumn{5}{|c|}{ Triglycerides $(\mathrm{mg} / \mathrm{dL})(\mathrm{n}=1,227)$} \\
\hline$<150$ & $787(64.1)$ & $316(40.2)$ & $107(33.9)$ & $209(66.1)$ \\
\hline$\geq 150$ & $440(35.9)$ & $251(57.0)$ & $132(52.6)$ & $119(47.4)$ \\
\hline$P$ value & & $<0.001$ & $<0.001$ & $<0.001$ \\
\hline \multicolumn{5}{|c|}{ Fasting glucose $(\mathrm{mg} / \mathrm{dL})(\mathrm{n}=1,226)$} \\
\hline$<126$ & $1,185(96.7)$ & $538(45.4)$ & $223(41.4)$ & $315(58.6)$ \\
\hline$\geq 126$ & $41(3.3)$ & $28(68.3)$ & $15(53.6)$ & $13(46.4)$ \\
\hline$P$ value & & 0.004 & 0.02 & 0.03 \\
\hline
\end{tabular}

Percentages for the column of total add $100 \%$; while for prevalence rates, the $100 \%$ is obtained adding the lines

Table 1: Characteristics of HIV-infected participants and prevalence of lipohypertrophy $[n(\%)]$.

obese, patients on HAART, with CD4 counts greater than 350 cells/ $\mathrm{mm}^{3}$ and undetectable viral load were significantly associated with the presence of lipohypertrophy. Increased levels of total cholesterol, HDL and LDL fractions, triglycerides and glucose were also associated with lipohypertropy.

When gender-related differences were analyzed, physical inactivity, obesity and undetectable viral load in men continued to be associated with lipohypertropy. Among women, those with non-white skin color, obesity, on HAART, CD4 counts greater than 350 cells $/ \mathrm{mm}^{3}$ and undetectable viral load were associated with lipohypertropy.

The characteristics of the participants, classified according to the presence or absence of lipoatrophy, are shown in table 2.

With regard to lipoatrophy, 660(53.2\%) had two or more signs of peripheral fat loss, and the percentage was nonsignificantly higher in men $(53.8 \%)$ than in women $(52.6 \%)$. Non-white skin color $(60.4 \%)$

\begin{tabular}{|c|c|c|c|c|}
\hline & \multirow{2}{*}{$\begin{array}{c}\text { Total } \\
(\mathrm{n}=1,240)\end{array}$} & \multicolumn{3}{|c|}{ Prevalence of Lipoatrophy } \\
\hline & & $\begin{array}{c}\text { Overall } \\
(n=1240)\end{array}$ & $\begin{array}{c}\text { Men } \\
(n=628)\end{array}$ & $\begin{array}{l}\text { Women } \\
(n=612)\end{array}$ \\
\hline \multicolumn{5}{|l|}{ Gender } \\
\hline Men & $628(50.6)$ & $338(53.8)$ & - & - \\
\hline Women & $612(49.4)$ & $322(52.6)$ & - & - \\
\hline$P$ value & & 0.7 & & \\
\hline \multicolumn{5}{|l|}{ Age (years) } \\
\hline $18-34$ & $471(38.0)$ & $240(51.0)$ & $98(40.8)$ & $142(59.2)$ \\
\hline $35-49$ & $591(47.7)$ & $329(55.7)$ & $191(58.0)$ & $138(42.0)$ \\
\hline $50-78$ & $178(14.3)$ & $91(51.1)$ & $49(53.8)$ & $42(46.2)$ \\
\hline$P$ value & & 0.3 & 0.2 & 0.4 \\
\hline \multicolumn{5}{|l|}{ Skin color } \\
\hline White & $692(55.8)$ & $329(47.5)$ & $178(54.1)$ & $151(45.9)$ \\
\hline Non-white & $548(44.2)$ & $331(60.4)$ & $160(48.3)$ & $171(51.7)$ \\
\hline$P$ value & & $<0.001$ & $<0.001$ & 0.033 \\
\hline \multicolumn{5}{|l|}{ Years at school } \\
\hline$<12$ & $1,097(88.5)$ & $606(55.2)$ & $305(50.3)$ & $301(49.7)$ \\
\hline$\geq 12$ & $143(11.5)$ & $54(37.8)$ & $33(61.1)$ & $21(38.9)$ \\
\hline$P$ value & & $<0.001$ & $<0.001$ & 0.3 \\
\hline \multicolumn{5}{|l|}{ Smoking (pack-years) } \\
\hline Never-smoker & $422(34.0)$ & $202(47.9)$ & $86(42.6)$ & $116(57.4)$ \\
\hline$<20$ & $498(40.2)$ & $265(53.2)$ & $128(48.3)$ & $137(51.7)$ \\
\hline$\geq 20$ & $320(25.8)$ & $193(60.3)$ & $124(64.2)$ & $69(35.8)$ \\
\hline$P$ value & & 0.003 & 0.04 & 0.10 \\
\hline \multicolumn{5}{|l|}{ Physical activity } \\
\hline Active & $716(57.7)$ & $397(55.4)$ & $188(47.4)$ & $209(52.6)$ \\
\hline Inactive or sedentary & $524(42.3)$ & $263(50.2)$ & $150(57.0)$ & $113(43.0)$ \\
\hline$P$ value & & 0.07 & 0.3 & 0.09 \\
\hline \multicolumn{5}{|c|}{ Binge drinking $(n=1,226)$} \\
\hline Yes & $211(17.0)$ & $101(47.9)$ & $71(70.3)$ & $30(29.7)$ \\
\hline No & $1,029(83.0)$ & $559(54.3)$ & $267(47.8)$ & $292(52.2)$ \\
\hline$P$ value & & 0.4 & 0.2 & 0.17 \\
\hline \multicolumn{5}{|c|}{ Body mass index $\left(\mathrm{kg} / \mathrm{m}^{2}\right)$} \\
\hline$<25$ & $708(57.1)$ & $445(62.9)$ & $252(56.6)$ & $193(43.4)$ \\
\hline $25-29.9$ & $380(30.6)$ & $152(40.0)$ & 74 (48.7) & $78(51.3)$ \\
\hline$\geq 30$ & $152(12.3)$ & $63(41.4)$ & $12(19.0)$ & $51(81.0)$ \\
\hline$P$ value & & $<0.001$ & $<0.001$ & 0.003 \\
\hline \multicolumn{5}{|c|}{ Years since HIV diagnosis } \\
\hline$<3$ & $485(39.1)$ & $234(48.2)$ & $113(48.3)$ & $121(51.7)$ \\
\hline $3-5$ & $350(28.3)$ & $174(49.7)$ & $88(50.6)$ & $86(49.4)$ \\
\hline$\geq 6$ & $404(32.6)$ & $252(62.4)$ & $137(54.4)$ & $115(45.6)$ \\
\hline$P$ value & & $<0.001$ & 0.002 & 0.02 \\
\hline \multicolumn{5}{|l|}{ Currently on HAART } \\
\hline Yes & $815(65.7)$ & $474(58.2)$ & $248(52.3)$ & $226(47.7)$ \\
\hline No & $425(34.3)$ & $186(43.8)$ & $90(48.4)$ & $96(51.6)$ \\
\hline
\end{tabular}


Citation: Trevisol FS, Alencastro PR, Ribeiro PAB, Wollf FH, Ikeda MLR, et al. (2012) Association of Physical Activity with Lipodystrophy Syndrome in HIV-Infected Patients. J AIDS Clinic Res 3:177. doi:10.4172/2155-6113.1000177

\begin{tabular}{|c|c|c|c|c|}
\hline$P$ value & & $<0.001$ & $<0.001$ & 0.002 \\
\hline \multicolumn{5}{|c|}{ CD4 count $\left(\mathrm{cel} / \mathrm{mm}^{3}\right)(\mathrm{n}=1,227)$} \\
\hline$<350$ & $476(38.8)$ & $265(55.7)$ & $148(55.8)$ & $117(44.2)$ \\
\hline$\geq 350$ & $751(61.2)$ & $387(51.5)$ & $185(47.8)$ & $202(52.2)$ \\
\hline$P$ value & & 0.16 & 0.04 & 0.9 \\
\hline \multicolumn{5}{|c|}{ HIV-RNA viral load $(n=1,221)$} \\
\hline Undetectable & $713(58.4)$ & $279(39.1)$ & $151(54.1)$ & $128(45.9)$ \\
\hline Detectable & $508(41.6)$ & $369(72.6)$ & $181(49.1)$ & $188(50.9)$ \\
\hline$P$ value & & 0.3 & 0.3 & 0.7 \\
\hline \multicolumn{5}{|c|}{ Total cholesterol $(\mathrm{mg} / \mathrm{dL})(\mathrm{n}=1,226)$} \\
\hline$<200$ & $854(69.7)$ & $465(54.4)$ & $242(52.0)$ & $223(48.0)$ \\
\hline$\geq 200$ & $372(30.3)$ & $186(50.0)$ & $89(47.8)$ & $97(52.2)$ \\
\hline$P$ value & & 0.04 & 0.13 & 0.15 \\
\hline \multicolumn{5}{|c|}{$\mathrm{HDL}(\mathrm{mg} / \mathrm{dL})(\mathrm{n}=1,226)$} \\
\hline$<35$ & $152(12.4)$ & $81(53.3)$ & $58(71.6)$ & $23(28.4)$ \\
\hline$\geq 35$ & $\begin{array}{c}1,074(87.6) \\
(86.7)\end{array}$ & $570(53.1)$ & $273(47.9)$ & $297(52.1)$ \\
\hline$P$ value & & 0.9 & 0.7 & 0.6 \\
\hline \multicolumn{5}{|c|}{$\operatorname{LDL}(\mathrm{mg} / \mathrm{dL})(\mathrm{n}=1,225)$} \\
\hline$<130$ & $966(78.9)$ & $528(54.7)$ & $280(53.0)$ & $248(47.0)$ \\
\hline$\geq 130$ & $259(21.1)$ & $122(47.1)$ & $50(41.0)$ & $72(59.0)$ \\
\hline$P$ value & & 0.03 & 0.19 & 0.09 \\
\hline \multicolumn{5}{|c|}{ Triglycerides $(\mathrm{mg} / \mathrm{dL})(\mathrm{n}=1,227)$} \\
\hline$<150$ & $787(64.1)$ & $419(53.2)$ & $203(48.4)$ & $216(51.6)$ \\
\hline$\geq 150$ & $440(35.9)$ & $232(52.7)$ & $128(55.2)$ & $104(44.8)$ \\
\hline$P$ value & & 0.9 & 0.10 & 0.15 \\
\hline \multicolumn{5}{|c|}{ Fasting glucose (mg/dL) (n=1,226) } \\
\hline$<126$ & $1,185(96.7)$ & $629(53.1)$ & $316(50.2)$ & $313(49.8)$ \\
\hline$\geq 126$ & $41(3.3)$ & $22(53.7)$ & $15(68.2)$ & $7(31.8)$ \\
\hline$P$ value & & 0.9 & 0.5 & 0.5 \\
\hline
\end{tabular}

Percentages for the column of total add $100 \%$; while for prevalence rates, the $100 \%$ is in the lines

Table 2: Characteristics of HIV-infected participants and prevalence of lipoatrophy.

and low education level (55.2\%) variables were significantly associated with lipoatrophy $(\mathrm{P}$ value $<0.001)$. Individuals classified as physically active, measured by the IPAQ, had higher prevalence of lipoatrophy (55.4\%, $\mathrm{P}$ value 0.07 ) than the others. Smoking ( $\mathrm{P}$ value 0.003 ) and $\mathrm{BMI}<25 \mathrm{~kg} / \mathrm{m}^{2}(\mathrm{P}$ value $<0.001)$ were also positively associated with the presence of lipoatrophy. In addition, HIV-infected individuals with longer diagnosis time and on HAART also had association with the presence of lipoatrophy.

Table 3 shows the association between physical activity and lipohypertropy and lipoatrophy in both men and women, adjusted for confounding factors modeled in a progressive way. Physical inactivity showed to be a risk factor for lipohypertropy only in men, being associated even after the full control for confounding factors.

Table 4 shows the association between physical activity measured by the IPAQ and mean fasting glucose and lipid levels. Metabolic parameters seem to be higher among individuals on HAART, in comparison to HAART-naive patients. Among individuals HAARTnaive, physically activity had lower mean total cholesterol, LDL and fasting glucose $(\mathrm{P}$ value $<0.03)$ than among insufficient active individuals, while for those on HAART, only triglycerides levels were higher among the insufficient active ( $\mathrm{P}$ value $=0.02$ ).

\section{Discussion}

This study detected high prevalence of lipohypertrophy (46\%) and lipoatrophy (53.2\%) among HIV-infected individuals. These results are in accordance with prospective studies, which were confirmed with the use of Dual X-Ray Absorptiometry (DEXA), high prevalence of both conditions. Prevalence of lipodystrophy ranged from 14\% to $63 \%$ [16]. There was an association between gender and lipohypertrophy, since it was more prevalent in women than in men. The findings of this study are consistent with those previously described, since women are at increased risk for central fat accumulation, whereas men have a higher risk for facial lipoatrophy [17]. However, this latter finding was not found in this study.

Lipodystrophy was also associated with non-white skin color. Even lipoatrophy has been associated with white skin color non-Whites seem to have higher central fat accumulation [18,19]. This finding

\begin{tabular}{|c|c|c|c|c|}
\hline \multirow[b]{2}{*}{ IPAQ } & \multicolumn{2}{|l|}{ Lipohypertrophy } & \multicolumn{2}{|l|}{ Lipoatrophy } \\
\hline & \begin{tabular}{|c|} 
Prevalence Ratio \\
$(95 \% \mathrm{Cl})$
\end{tabular} & $\begin{array}{c}\mathrm{P} \\
\text { value }\end{array}$ & $\begin{array}{c}\text { Prevalence Ratio } \\
(95 \% \mathrm{Cl})\end{array}$ & $\begin{array}{c}\mathrm{P} \\
\text { value }\end{array}$ \\
\hline $\begin{array}{l}\text { Men } \\
\text { Actives* } \\
\text { Insufficient physical } \\
\text { activity }\end{array}$ & $\begin{array}{l}1.0 \\
1.31(1.07-1.60)\end{array}$ & 0.008 & $\begin{array}{l}1.0 \\
0.93(0.80-1.08)\end{array}$ & 0.3 \\
\hline Model $1^{*}$ & $1.31(1.07-1.59)$ & 0.008 & $0.93(0.80-1.07)$ & 0.3 \\
\hline Model $2^{* *}$ & $1.30(1.07-1.59)$ & 0.009 & $0.94(0.91-1.08)$ & 0.4 \\
\hline Model $3^{* * *}$ & $1.30(1.07-1.58)$ & 0.01 & $0.94(0.82-1.09)$ & 0.4 \\
\hline Model $4^{\star * \star *}$ & $1.30(1.07-1.58)$ & 0.009 & $0.94(0.82-1.09)$ & 0.4 \\
\hline Model $5^{\star \star \star \star \star}$ & $1.34(1.10-1.63)$ & 0.004 & $0.93(0.80-1.07)$ & 0.3 \\
\hline Model $6^{\star \star * * * *}$ & $1.34(1.10-1.63)$ & 0.004 & $0.92(0.80-1.07)$ & 0.3 \\
\hline $\begin{array}{l}\text { Women } \\
\text { Actives* } \\
\text { Insufficient physical } \\
\text { activity }\end{array}$ & $\begin{array}{l}1.0 \\
0.91(0.78-1.06)\end{array}$ & 0.2 & $\begin{array}{l}1.0 \\
0.87(0.74-1.03)\end{array}$ & 0.10 \\
\hline Model 1* & $0.91(0.78-1.06)$ & 0.2 & $0.88(0.75-1.03)$ & 0.11 \\
\hline Model $2^{* *}$ & $0.92(0.79-1.08)$ & 0.3 & $0.89(0.76-1.04)$ & 0.15 \\
\hline Model $3^{* * *}$ & $0.93(0.79-1.08)$ & 0.3 & $0.89(0.76-1.05)$ & 0.17 \\
\hline Model $4^{\star \star \star \star}$ & $0.92(0.79-1.08)$ & 0.3 & $0.90(0.76-1.06)$ & 0.19 \\
\hline Model $5^{\star \star \star \star *}$ & $0.93(0.80-1.08)$ & 0.3 & $0.90(0.77-1.06)$ & 0.2 \\
\hline Model $6^{\star * \star * * *}$ & $0.93(0.80-1.08)$ & 0.3 & $0.91(0.77-1.07)$ & 0.2 \\
\hline
\end{tabular}

*adjusted for age

**adjusted for age and race

***adjusted for age, race and education

****adjusted for age, race, education and pack years

*****adjusted for age, race, education, binge drinking and CD4

adjusted for age, race, education, binge drinking, CD4 and HAART

Table 3: Association between physical activity and lipohypertrophy and lipoatrophy adjusted for confounding variables.

\begin{tabular}{|c|c|c|c|c|}
\hline & Mean (SD) & $\begin{array}{l}\text { Insufficient } \\
\text { active }\end{array}$ & Active & P-value \\
\hline & \multicolumn{3}{|c|}{ HIV patients HAART-naïve $(n=425)$} & \\
\hline Total cholesterol (mg/dL) & $173.7 \pm 37.5$ & $179.6 \pm 38.0$ & $169.8 \pm 36.7$ & 0.009 \\
\hline $\mathrm{HDL}(\mathrm{mg} / \mathrm{dL})$ & $49.8 \pm 13.1$ & $50.3 \pm 12.2$ & $49.5 \pm 13.7$ & 0.5 \\
\hline $\mathrm{LDL}(\mathrm{mg} / \mathrm{dL})$ & $99.9 \pm 32.7$ & $104.6 \pm 34.0$ & $96.7 \pm 31.5$ & 0.02 \\
\hline Fasting glucose (mg/dL) & $84.3 \pm 22.3$ & $87.3 \pm 29.1$ & $82.3 \pm 16.0$ & 0.03 \\
\hline \multirow[t]{2}{*}{ Triglycerides (mg/dL) } & $120.6 \pm 63.3$ & $121.0 \pm 59.2$ & $120.3 \pm 66.0$ & 0.9 \\
\hline & \multicolumn{4}{|c|}{ HIV patients currently on HAART $(n=815)$} \\
\hline Total cholesterol (mg/dL) & $187.9 \pm 45.7$ & $189.5 \pm 44.9$ & $186.7 \pm 46.4$ & 0.4 \\
\hline $\mathrm{HDL}(\mathrm{mg} / \mathrm{dL})$ & $51.9 \pm 14.1$ & $51.1 \pm 14.6$ & $52.4 \pm 13.7$ & 0.2 \\
\hline LDL (mg/dL) & $103.0 \pm 38.0$ & $102.8 \pm 36.5$ & $103.2 \pm 39.1$ & 0.9 \\
\hline Fasting glucose (mg/dL) & $88.2 \pm 29.9$ & $88.9 \pm 30.1$ & $87.6 \pm 29.8$ & 0.5 \\
\hline Triglycerides (mg/dL) & $167.9 \pm 121.1$ & $179.1 \pm 122.2$ & $159.4 \pm 119.7$ & 0.02 \\
\hline
\end{tabular}

Table 4: Association between physical activity and metabolic profile among treatment-naive HIV-infected individuals or under antiretroviral therapy. 
is consistent with that described in the literature $[17,20,21]$ and may represent the presence of mixed lipodystrophy.

Lipohypertrophy was also associated with the use of HAART, CD4 count $\geq 350$ cells $/ \mathrm{mm}^{3}$ and undetectable viral load. In the gender-related analysis, the association of lipohypertrophy with these clinical variables was observed in women, but remained associated only with undetectable viral load in men. Longer HIV infection and the use of HAART were also positively associated with the presence of lipoatrophy. The literature shows some evidence of the association between antiretroviral treatment and the occurrence of lipodystrophy, since the introduction of HAART [22,23]. The factors most strongly associated with lipodystrophy are the use of HAART for longer period, use of protease inhibitors and age $[19,24,25]$. In this study, no analysis was performed in the use of HAART by class of antiretroviral drugs, but protease inhibitors are widely used in triple therapy in patients with clinical indication for using pharmacological treatment. Non-smokers had higher prevalence of lipohypertrophy, but this was not observed in the gender-related analysis, whereas smokers were associated with the presence of lipoatrophy. A study conducted with 450 male smokers, individuals not infected with HIV, found an association between smoking and increased visceral fat accumulation [26]. In the short term, nicotine increases energy expenditure and could reduce appetite, which may explain less weight gain than nonsmokers. However, smoking increases insulin resistance and is associated with central fat accumulation [26]. Obesity was directly associated with the presence of lipohypertrophy, and normal individuals were associated with lipoatrophy. Weight, height and BMI are important variables for lipodystrophy assessment. However, these criteria alone are not sufficient to distinguish patients with abnormal fat redistribution (lipoatrophy or lipohypertrophy), since they may be within normal limits in patients with mixed lipodystrophy [2].

This study showed that lipoatrophy was observed in a greater proportion among physically active individuals, but in the genderrelated analysis, there was no statistically significant difference even after adjustment for confounding variables. Lipohypertrophy in men was associated with inactivity, independently of age, skin color, education, binge drinking, CD4 and HAART. Such association was not observed among women. Few studies have analyzed the association of physical activity with HIV-lipodystrophy syndrome. A study conducted with $150 \mathrm{HIV}$-infected individuals taking Stavudine or Zidovudine concluded that physical activity is an independent protective factor for HIV-lipodystrophy syndrome, especially when combined with a proper diet [27]. Segatto et al. concluded that a physically active lifestyle has a protective effect against the occurrence of lipodystrophy related to HAART [28]. Physical activity is considered a preventive intervention for lipohypertrophy, which accounts for maintaining the body composition [29] and prevent the accumulation of central body fat [30]. However, despite the beneficial effect of exercise and/or physical activity in preventing lipohypertrophy [31], they may worsen lipoatrophy [6,12].

With regard to the lipid profile, in the whole sample, $35.5 \%$ of participants showed increased levels of triglycerides, $30 \%$ of total cholesterol, $20.9 \%$ of LDL fraction, 3.3\% of fasting glucose, and $12.3 \%$ had a reduced level of HDL. There was an association between subjects with lipohypertrophy and elevated levels of total cholesterol, HDL and LDL fractions, triglycerides and fasting glucose. Among women, only elevated levels of HDL and LDL fractions were not associated with lipohypertrophy. The use of hypotriglycemic drugs showed no difference in the prevalence of lipodystrophy (data not shown).
HIV-infected individuals have shown lipid and glucose abnormalities, usually associated with the use of HAART $[6,22,32,33]$. In a cohort of HIV-infected individuals in Uganda, there was an increase of basal levels of total cholesterol, HDL, LDL and triglycerides 24 months after starting HAART. Triglycerides returned to basal levels at the end of the follow-up period. In this case, the increase in HDL has been described as resulting from the increase in total cholesterol [34], as observed in this study. In addition, individuals who discontinued HAART had a reduction of lipid levels, and in the resumption of treatment, there was an increase in glucose and lipid levels in only eight weeks $[35,36]$. Change in triglyceride levels in HIV-infected individuals is a very common finding, regardless of use of HAART [37]. However, many authors consider hypercholesterolemia and hypertriglyceridemia associated with the use of protease inhibitors $[38,39]$.

Elevated serum triglyceride levels are associated with the occurrence of coronary heart disease $[40,41]$. Other changes in the lipid profile are also considered risk factors for cardiovascular disease, both in the general population and in HIV-infected individuals [7,42]. Despite the cardiovascular risk associated with the use and duration of HAART, there is evidence that chronic infection, inflammation and immune function imbalance, caused by HIV infection, contribute to changing the structure and vascular function $[43,44]$.

Physically active HAART-naive individuals had significantly lower levels of total cholesterol, LDL fraction and glucose. Among HAARTtreated subjects, physical activity was associated with lower levels of triglycerides. In adults without HIV infection, several studies have shown that exercise and physical activity have a protective effect on dyslipidemia and is associated with lower levels of total cholesterol, LDL fraction, and in particular, with lower value in serum triglyceride levels $[40,45,46]$.

The results of this study showed higher serum cholesterol, HDL, LDL, triglycerides and glucose among insufficient active individuals. This finding may indicate that the benefits of physical activity are also applicable to HIV-infected individuals, to reduce risk factors for cardiovascular disease. However, there are limitations of the experimental design, since cross-sectional studies cannot establish cause and effect relationship. The lipid profile is influenced by intrinsic factors such as individual metabolism and family history of dyslipidemia and diabetes (genetic) [47] and extrinsic factors such as diet $[48,49]$ and medication use $[50,51]$.

In conclusion, the high prevalence of lipohypertrophy among HIV-infected individuals was independently associated with inactivity, and clinical and behavioral characteristics could increase the risk by $34 \%$. Physically active HAART-naïve subjects had lower levels of total cholesterol, LDL fraction and fasting glucose levels, while those on HAART only had a significant reduction in triglyceride levels. Physical activity is a non-pharmacological strategy appropriate for the prevention or treatment of HIV lipodystrophy syndrome, whereas it has beneficial effects on lipohypertrophy and lipid levels.

\section{Acknowledgements}

This study was supported by grants and scholarships from the CNPq (National Council for Scientific and Technological Development), CAPES (Coordination for the Improvement of Higher Education Personnel), Fundação de Amparo a Pesquisa do Rio Grande do Sul (FAPERGS), and FIPE-HCPA (Fundo de Apoio a Pesquisa, Hospital de Clínicas de Porto Alegre)

The sponsors did not take part in the design or conduct of the study, including data collection, management, analysis, and interpretation of the data; and preparation, review, or approval of the manuscript.

The authors had full access to all of the data in the study and take responsibility for the integrity of the data and the accuracy of the data analysis. 
Citation: Trevisol FS, Alencastro PR, Ribeiro PAB, Wollf FH, Ikeda MLR, et al. (2012) Association of Physical Activity with Lipodystrophy Syndrome in HIV-Infected Patients. J AIDS Clinic Res 3:177. doi:10.4172/2155-6113.1000177

\section{References}

1. Palella FJ Jr, Delaney KM, Moorman AC, Loveless MO, Fuhrer J, et al. (1998) Declining morbidity and mortality among patients with advanced human immunodeficiency virus infection. HIV Outpatient Study Investigators. N Engl J Med 338: 853-860.

2. Baril JG, Junod P, Leblanc R, Dion H, Therrien R, et al. (2005) HIV-associated lipodystrophy syndrome: A review of clinical aspects. Can J Infect Dis Med Microbiol 16: 233-243.

3. Kravcik S (2000) HIV lipodystrophy: a review. HIV Clin Trials 1: 37-50.

4. Bodasing N, Fox R (2003) HIV-associated lipodystrophy syndrome: assessment and management. J Infect 46: 87-93.

5. Constans J, Pellegrin JL, Peuchant E, Dumon MF, Pellegrin I, et al. (1994) Plasma lipids in HIV-infected patients: a prospective study in 95 patients. Eur $\mathrm{J}$ Clin Invest 24: 416-420.

6. Carr A (2003) HIV lipodystrophy: risk factors, pathogenesis, diagnosis and management. AIDS 17 : S141-148.

7. Bergersen BM, Sandvik L, Bruun JN, Tonstad S (2004) Elevated Framingham risk score in HIV-positive patients on highly active antiretroviral therapy: results from a Norwegian study of 721 subjects. Eur J Clin Microbiol Infect Dis 23: 625-630

8. Collins RL, Kanouse DE, Gifford AL, Senterfitt JW, Schuster MA, et al. (2001) Changes in health-promoting behavior following diagnosis with HIV: prevalence and correlates in a national probability sample. Health Psychol 20: 351-360.

9. Thoni GJ, Fedou C, Brun JF, Fabre J, Renard E, et al. (2002) Reduction of fat accumulation and lipid disorders by individualized light aerobic training in human immunodeficiency virus infected patients with lipodystrophy and/or dyslipidemia. Diabetes Metab 28: 397-404

10. Terry L (2008) HIV and exercise. Journal of the Society of Cardiology of Rio Grande do Sul $15: 1-7$.

11. Terry L, Sprinz E, Stein R, Medeiros NB, Oliveira J, et al. (2006) Exercise training in HIV-1-infected individuals with dyslipidemia and lipodystrophy. Med Sci Sports Exerc 38: 411-417.

12. Roubenoff R, Weiss L, McDermott A, Heflin T, Cloutier GJ, et al. (1999) A pilot study of exercise training to reduce trunk fat in adults with HIV-associated fat redistribution. AIDS 13: 1373-1375.

13. Azevedo MR, Araújo CL, Reichert FF, Siqueira FV, da Silva MC, et al. (2007) Gender differences in leisure-time physical activity. Int J Public Health 52: 8-15.

14. Jaime PC, Florindo AA, Latorre Mdo R, Segurado AA (2006) Central obesity and dietary intake in HIVIAIDS patients. Rev Saude Publica 40: 634-640.

15. Cui Q, Carruthers S, Mclvor A, Smaill F, Thabane L, et al. (2010) Effect of smoking on lung function, respiratory symptoms and respiratory diseases amongst HIV-positive subjects: a cross-sectional study. AIDS Res Ther 7: 6 .

16. Peterson S, Martins CR, Cofrancesco J Jr (2008) Lipodystrophy in the patien with HIV: social, psychological, and treatment considerations. Aesthet Surg J 28: 443-451.

17. Lichtenstein KA, Delaney KM, Armon C, Ward DJ, Moorman AC, et al. (2003) Incidence of and risk factors for lipoatrophy (abnormal fat loss) in ambulatory HIV-1-infected patients. J Acquir Immune Defic Syndr 32: 48-56.

18. Bogner JR, Vielhauer V, Beckmann RA, Michl G, Wille L, et al. (2001) Stavudine versus zidovudine and the development of lipodystrophy. J Acquir Immune Defic Syndr 27: 237-244.

19. Andany N, Raboud JM, Walmsley S, Diong C, Rourke SB, et al. (2011) Ethnicity and gender differences in lipodystrophy of HIV-positive individuals taking antiretroviral therapy in Ontario, Canada. HIV Clin Trials 12: 89-103.

20. Galli M, Veglia F, Angarano G, Santambrogio S, Meneghini E, et al. (2003) Gender differences in antiretroviral drug-related adipose tissue alterations Women are at higher risk than men and develop particular lipodystrophy patterns. J Acquir Immune Defic Syndr 34: 58-61.

21. Jacobson DL, Knox T, Spiegelman D, Skinner S, Gorbach S, et al. (2005) Prevalence of, evolution of, and risk factors for fat atrophy and fat deposition in a cohort of HIV-infected men and women. Clin Infect Dis 40: 1837-1845.

22. Carr A, Cooper DA (2000) Adverse effects of antiretroviral therapy. Lancet 356: 1423-1430.
23. Carr A (2000) HIV protease inhibitor-related lipodystrophy syndrome. Clin Infect Dis 30: $\mathrm{S} 135-142$

24. Miller J, Carr A, Emery S, Law M, Mallal S, et al. (2003) HIV lipodystrophy: prevalence, severity and correlates of risk in Australia. HIV Med 4: 293-301.

25. Chen D, Misra A, Garg A (2002) Clinical review 153: Lipodystrophy in human immunodeficiency virus-infected patients. J Clin Endocrinol Metab 87: 48454856 .

26. Komiya H, Mori Y, Yokose T, Tajima N (2006) Smoking as a risk factor for visceral fat accumulation in Japanese men. Tohoku J Exp Med 208: 123-132.

27. Domingo P, Sambeat MA, Perez A, Ordonez J, Rodriguez J, et al. (2003) Fat distribution and metabolic abnormalities in HIV-infected patients on firs combination antiretroviral therapy including stavudine or zidovudine: role of physical activity as a protective factor. Antivir ther 8: 223-231.

28. Segatto AF, Freitas Junior IF, Santos VR, Alves KC, Barbosa DA, et al. (2011) Lipodystrophy in HIVIAIDS patients with different levels of physical activity while on antiretroviral therapy. Rev Soc Bras Med Trop 44: 420-424.

29. Ramirez-Marrero FA, Smith BA, Melendez-Brau N, Santana-Bagur JL (2004) Physical and leisure activity, body composition, and life satisfaction in HIVpositive Hispanics in Puerto Rico. J Assoc Nurses AIDS Care 15: 68-77.

30. Florindo AA, de Oliveira Latorre Mdo R, Jaime PC, Segurado AA (2007) Leisure time physical activity prevents accumulation of central fat in HIVIAIDS subjects on highly active antiretroviral therapy. Int J STD AIDS 18: 692-696.

31. Smith BA, Neidig JL, Nickel JT, Mitchell GL, Para MF, et al. (2001) Aerobic exercise: effects on parameters related to fatigue, dyspnea, weight and body composition in HIV-infected adults. AIDS 15: 693-701.

32. Carr A, Samaras K, Burton S, Law M, Freund J, et al. (1998) A syndrome of peripheral lipodystrophy, hyperlipidaemia and insulin resistance in patients receiving HIV protease inhibitors. AIDS 12: F51-58.

33. Guimaraes MM, Greco DB, de O Junior AR, Penido MG, Machado LJ (2007) Corporal fat distribution and lipidic and glicemic profiles of HIV-infected patients. Arq Bras Endocrinol Metabol 51: 42-51.

34. Buchacz K, Weidle PJ, Moore D, Were W, Mermin J, et al. (2008) Changes in lipid profile over 24 months among adults on first-line highly active antiretrovira therapy in the home-based AIDS care program in rural Uganda. J Acquir Immune Defic Syndr 47: 304-311.

35. Seoane E, Resino S, Micheloud D, Moreno A, de Quiros JC, et al. (2008) Lipid and apoprotein profile in HIV-1-infected patients after CD4-guided treatment interruption. J Acquir Immune Defic Syndr 48: 455-459.

36. Skiest DJ, Krambrink A, Su Z, Robertson KR, Margolis DM (2008) Improved measures of quality of life, lipid profile, and lipoatrophy after treatment interruption in HIV-infected patients with immune preservation: results of ACTG 5170. J Acquir Immune Defic Syndr 49: 377-383.

37. Grunfeld C, Feingold KR (1992) Metabolic disturbances and wasting in the acquired immunodeficiency syndrome. N Engl J Med 327: 329-337.

38. Tsiodras S, Mantzoros C, Hammer S, Samore M (2000) Effects of protease inhibitors on hyperglycemia, hyperlipidemia, and lipodystrophy: a 5-year cohort study. Arch Intern Med 160: 2050-2056.

39. Heath KV, Chan KJ, Singer J, O'Shaughnessy MV, Montaner JS, et al. (2002) Incidence of morphological and lipid abnormalities: gender and treatment differentials after initiation of first antiretroviral therapy. Int $\mathrm{J}$ Epidemiol 31: 1016-1020.

40. Tirosh A, Rudich A, Shochat T, Tekes-Manova D, Israeli E, et al. (2007) Changes in triglyceride levels and risk for coronary heart disease in young men. Ann Intern Med 147: 377-385.

41. Sarwar N, Danesh J, Eiriksdottir G, Sigurdsson G, Wareham N, et al. (2007) Triglycerides and the risk of coronary heart disease: 10,158 incident cases among 262,525 participants in 29 Western prospective studies. Circulation 115: $450-458$.

42. Baum MK, Rafie C, Lai S, Xue L, Sales S, et al. (2006) Coronary Heart Disease (CHD) Risk Factors and Metabolic Syndrome in HIV-Positive Drug Users in Miami. Am J Infect Dis 2: 173-179.

43. Boccara F (2008) Cardiovascular complications and atherosclerotic manifestations in the HIV-infected population: type, incidence and associated risk factors. AIDS 22: S19-26. 
Citation: Trevisol FS, Alencastro PR, Ribeiro PAB, Wollf FH, Ikeda MLR, et al. (2012) Association of Physical Activity with Lipodystrophy Syndrome in HIV-Infected Patients. J AIDS Clinic Res 3:177. doi:10.4172/2155-6113.1000177

44. Fichtenbaum CJ (2010) Does antiretroviral therapy increase or decrease the risk of cardiovascular disease? Curr HIVIAIDS Rep 7: 92-98.

45. Guedes DP, Goncalves LA (2007) Impact of the habitual physical activity on lipid profile in adults. Arq Bras Endocrinol Metabol 51: 72-78.

46. Healy GN, Dunstan DW, Salmon J, Cerin E, Shaw JE, et al. (2008) Breaks in sedentary time: beneficial associations with metabolic risk. Diabetes Care 31: 661-666

47. Yamada Y, Matsuo H, Warita S, Watanabe S, Kato K, et al. (2007) Prediction of genetic risk for dyslipidemia. Genomics 90: 551-558.
48. Arendt BM, Aghdassi E, Mohammed SS, Fung LY, Jalali P, et al. (2008) Dietary intake and physical activity in a Canadian population sample of male patients with HIV infection and metabolic abnormalities. Curr HIV Res 6: 82-90.

49. Sheehan LA, Macallan DC (2000) Determinants of energy intake and energy expenditure in HIV and AIDS. Nutrition 16: 101-106.

50. (2002) Third Report of the National Cholesterol Education Program (NCEP) Expert Panel on Detection, Evaluation, and Treatment of High Blood Cholestero in Adults (Adult Treatment Panel III) final report. Circulation 106: 3143-3421.

51. (2005) I Brazilian guidelines on diagnosis and treatment of metabolic syndrome. Arq Bras Cardiol 84: 1-28. 